\title{
Optimization of Highly Noncoplanar Arc Therapy Trajectories: a Dosimetric Approach
}

\author{
Humberto Rocha ${ }^{1,2}$, Joana Dias ${ }^{1,2}$, Tiago Ventura ${ }^{2,3}$, \\ Brígida Ferreira ${ }^{2,4}$, and Maria do Carmo Lopes ${ }^{2,3}$ \\ 1 CeBER and FEUC, Universidade de Coimbra, 3004-512 Coimbra, Portugal \\ 2 INESC-Coimbra, 3030-290 Coimbra, Portugal \\ 3 Serviço de Física Médica, IPOC-FG, EPE, 3000-075 Coimbra, Portugal \\ 4 Escola Superior de Saúde, Politécnico do Porto 4200-072 Porto, Portugal \\ hrocha@mat.uc.pt, joana@fe.uc.pt, tiagoventura@ipocoimbra.min-saude.pt, \\ bcf@ess.ipp.pt, mclopes@ipocoimbra.min-saude.pt
}

\begin{abstract}
The latest generation of linear accelerators allows the use of noncoplanar trajectories in arc therapy which combine the benefits of noncoplanar intensity-modulated radiation therapy (IMRT) treatment plans, such as improved organ sparing, with the benefits of arc therapy treatment plans, such as short treatment times. In this paper, we propose a two-step approach based on dosimetric criteria for the optimization of noncoplanar arc trajectories. In the first step, an initial set of anchor points (noncoplanar beam directions) is computed using a beam angle optimization (BAO) algorithm. In the second step, anchored in the beam directions already calculated, the noncoplanar arc trajectory is defined by iteratively computing additional anchor points considering the same dosimetric criteria used for the noncoplanar BAO. A nasopharyngeal tumor case already treated at the Portuguese Institute of Oncology of Coimbra (IPOC), is used to illustrate the benefits of the proposed optimization approach.
\end{abstract}

Keywords: radiation therapy, noncoplanar arc therapy, optimization

\section{Introduction}

In step-and-shoot IMRT, a linear accelerator mounted on a gantry rotates around the patient, stops at fixed beam directions and delivers non-uniform radiation fields. Noncoplanar beam directions are obtained if the couch is allowed to rotate as well. In arc therapy, irradiation is done continuously while the gantry rotates around the patient with the treatment beam always on. One of the most efficient IMRT arc techniques is volumetric modulated arc therapy (VMAT), particularly in terms of dose delivery time [1-3]. VMAT treatment plans typically use coplanar trajectories, considering a fixed couch angle equal to $0^{\circ}$.

The latest generation of linear accelerators allows the use of noncoplanar trajectories in arc therapy which combine the benefits of noncoplanar IMRT treatment plans, such as improved organ sparing, with the benefits of arc therapy 
treatment plans, such as short treatment times. Several authors have proposed approaches to optimize highly noncoplanar arc trajectories, mainly resorting to geometrical metrics $[5,6]$.

In this paper, we propose a two-step approach based on dosimetric criteria for the optimization of noncoplanar arc trajectories. In the first step, an initial set of anchor points (noncoplanar beam directions) is computed taking advantage of the work previously developed in BAO for step-and-shoot IMRT [7-13]. In the second step, anchored in the beam directions already calculated, the noncoplanar arc trajectory is defined by iteratively computing additional anchor points considering the same dosimetric criteria used for the noncoplanar BAO. A nasopharyngeal tumor case already treated at IPOC is used to illustrate the benefits of the proposed approach. The paper is organized as follows. The strategy proposed for noncoplanar arc trajectory optimization is described in the next Section. In Section three we present the computational results. Conclusions and future work are presented in the last Section.

\section{Noncoplanar Arc Trajectory Optimization}

In this study, simultaneous gantry and couch rotation is considered while the treatment beam is on, leading to a highly noncoplanar arc trajectory. An optimization approach for the noncoplanar arc trajectory of a VMAT plan, called $n c V M A T$, is proposed and compared with the coplanar arc trajectory of a VMAT plan, called $c V M A T$, and with the typically used equispaced step-and-shoot IMRT plan, called Equi. The two-step approach proposed combines two optimization problems, the $\mathrm{BAO}$ problem and the arc trajectory optimization, that are quite challenging just by themselves. The dosimetric criteria used to guide these two optimization problems is the optimal value of the fluence map optimization (FMO) problem. Formulation and resolution approaches used to address FMO and BAO problems correspond to the ones detailed in Rocha et al. $[12]$.

A clinical nasopharyngeal tumor case treated at IPOC was used to illustrate and test our approach. Nasopharyngeal tumors are complex cases to plan due to the large number of organs-at-risk (OARs) in the neighborhood of the tumor(s). The spinal cord and the brainstem are two of the main OARs considered. They are serial organs, i.e, they are compromised even if only a small part is damaged. Therefore, maximum-doses are prescribed for spinal cord and brainstem, $45 \mathrm{~Gy}$ and 54 Gy respectively. The other OARs considered are the parotids (the larger salivary glands) and the oral cavity (that contains the remaining salivary glands). These are parallel organs whose functioning is not much affected if a small part of the organ is damaged. Thus, mean-doses are prescribed for parotids and oral cavity, 26 Gy and 45 Gy respectively. For safety purposes, the tumor volume is enlarged by adding a margin originating a structure called planning target volume (PTV). Two levels of radiation dose are prescribed: a higher radiation dose of 70 Gy is prescribed to the tumor (called $P T V_{70}$ ) and a lower radiation dose of 59.4 Gy is prescribed to the lymph nodes (called $P T V_{59.4}$ ). 


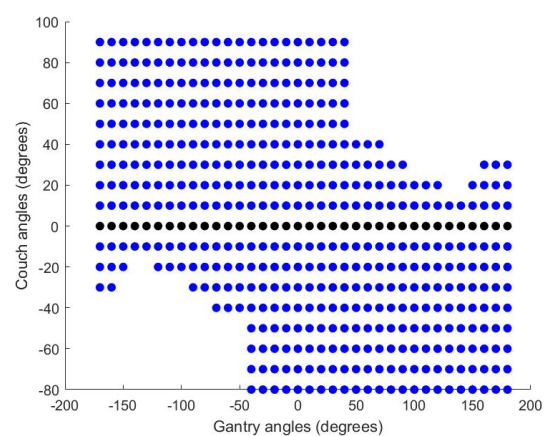

(a)

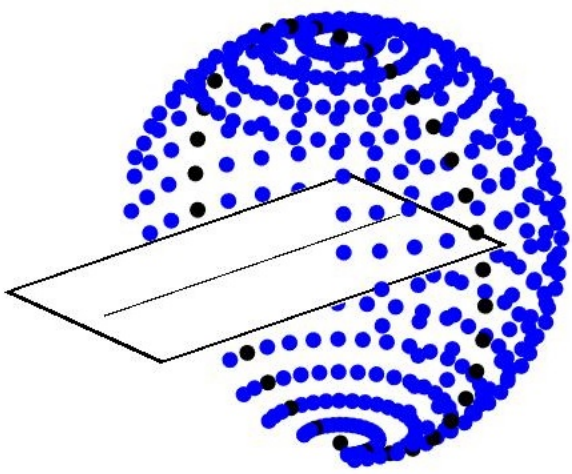

(b)

Fig. 1. Candidate beams homogeneously distributed represented in $2 \mathrm{D}-1$ (a) and the corresponding $3 \mathrm{D}$ representation $-1(\mathrm{~b})$. Black beams correspond to the coplanar candidate beams (couch fixed at $0^{\circ}$ ) while blue beams correspond to noncoplanar candidate beams.

The second step, arc trajectory optimization, is now described. A grid with equispaced beams, separated by $10^{\circ}$ for both the gantry and the couch, is considered in this second step. After exclusion of infeasible couch-gantry angle pairs due to possible collisions of patient and gantry for a nasopharyngeal tumor case, we end up with 472 candidate beams homogeneously distributed as illustrated in Fig. 1.

The initial anchor points corresponding to the 7-beam noncoplanar BAO solution for the nasopharyngeal tumor case at hand are displayed in red in Fig. 2(a). In order to enhance one of the main features of VMAT, short treatment times, the arc trajectory starts at the leftmost anchor point in Fig. 2(a), visit each anchor point from left to right and ends at the rightmost anchor point in Fig. 2(a), with the gantry always rotating towards the next anchor point while the couch might be halted or moving towards the next anchor point. Considering these gantry/couch movement restrictions, the feasible points when calculating a new anchor point are shown in green in Fig. 2(a).

There are different ways of considering the optimal value of the FMO problem to iteratively add novel anchor points, one by one. In this study we consider the most expensive, in terms of computational time, that adds each one of the green points, one at a time, to the existing set of anchor points and then compute the corresponding optimal FMO value considering these beams. The candidate beam that leads to the minimum optimal FMO value when added to the existing anchor beams will be selected as the next anchor point. In Fig. 2(b) the novel anchor point is displayed. This recently added red point leads to the infeasibility of some green beams due to the gantry/couch movements defined. At the end of each iteration, green candidate beams that became infeasible are removed as illustrated in Fig. 2(b). This iterative procedure ends when 20 anchor points 


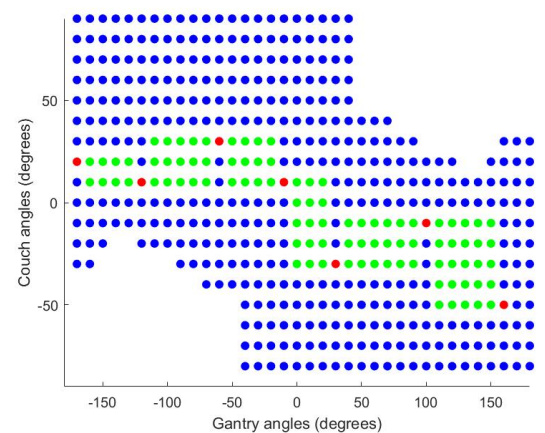

(a)

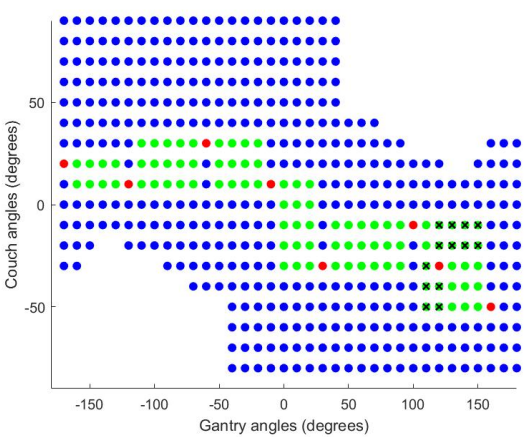

(b)

Fig. 2. The 7-beam noncoplanar BAO solution is displayed in red and the feasible points to consider when calculating a new anchor point are displayed in green $-2(\mathrm{a})$. A novel anchor point is added and green candidate beams that became infeasible are removed $-2(b)$.

Table 1. Target coverage, conformity and homogeneity obtained by treatment plans.

\begin{tabular}{clccc}
\hline Target parameters & Equi & $c$ VMAT & ncVMAT \\
\hline \multirow{2}{*}{ Coverage } & 0.877 & 0.882 & 0.888 \\
Conformity & 0.556 & 0.523 & 0.550 \\
& Homogeneity & 0.880 & 0.881 & 0.881 \\
Coverage & 0.907 & 0.911 & 0.880 \\
CTV 59.4 Conformity & 0.784 & 0.760 & 0.841 \\
Homogeneity & 0.774 & 0.773 & 0.767 \\
\hline
\end{tabular}

are obtained, which is the typical number of anchor points considered in the literature (see, e.g., [14, 15]).

\section{Computational Results}

Computational tests were conducted on a Dell Precision T5600 with Intel Xeon processor $64 \mathrm{~GB} 1600 \mathrm{MHz}$. The noncoplanar arc trajectory obtained is displayed in Fig. 3. In terms of optimal FMO values, ncVMAT clearly outperforms the other treatment plans in terms of optimal FMO value, improving $9.8 \%$ the value obtained by Equi plan while the improvement of $c V M A T$ was $5.5 \%$. For a similar tumor volume coverage, displayed in Table 1, the organ sparing, reported in Table 2, also shows the advantage of the $n c V M A T$ treatment plan. 


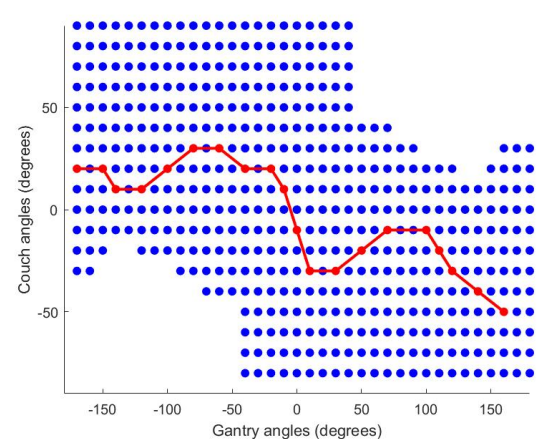

(a)

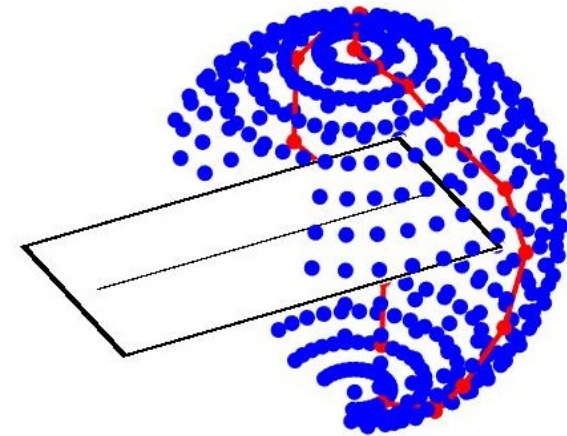

(b)

Fig. 3. Trajectory obtained by the noncoplanar arc trajectory optimization approach in $2 \mathrm{D}-3(\mathrm{a})$ and in $3 \mathrm{D}-3(\mathrm{~b})$

Table 2. OARs sparing obtained by treatment plans.

\begin{tabular}{lcccccccc}
\hline & \multicolumn{3}{c}{ Mean Dose (Gy) } & & \multicolumn{3}{c}{ Max Dose (Gy) } \\
\cline { 2 - 3 } \cline { 7 - 8 } OAR & Equi & $c V M A T$ & ncVMAT & & Equi & cVMAT & ncVMAT \\
\hline Spinal cord & - & - & - & & 33.4 & 29.1 & 33.1 \\
Brainstem & - & - & - & & 52.1 & 49.5 & 43.4 \\
Right parotid & 37.0 & 34.8 & 34.5 & & - & - & - \\
Left parotid & 32.0 & 29.8 & 27.6 & & - & - & - \\
Oral Cavity & 26.2 & 22.3 & 22.4 & & - & - & - \\
\hline
\end{tabular}

\section{Conclusions and Future Work}

A dosimetric approach for the optimization of highly noncoplanar arc trajectories was described and tested using a complex nasopharyngeal tumor case already treated at IPOC. For the patient tested, the resulting noncoplanar arc plan, $n c V$ $M A T$, clearly outperforms both the coplanar arc plan, $c V M A T$, and the typically used coplanar equispaced step-and-shoot IMRT plan. Although, for the patient at hand, the overall quality of the treatment is undoubtedly greater considering the noncoplanar arc plan, tests with more patients should be conducted. Moreover, other strategies to accelerate both the noncoplanar BAO procedure and the second step that determines the remaining anchor points should be investigated.

\section{Acknowledgments}

This work has been supported by project grant POCI-01-0145-FEDER-028030 and by the Fundação para a Ciência e a Tecnologia (FCT) under project grant UID/Multi/00308/2019. 
Humberto Rocha et al.

\section{Conflicts of Interest}

None.

\section{References}

1. Bedford, J. L.: Treatment planning for volumetric modulated arc therapy. Med. Phys. 36, 5128-5138 (2009)

2. Otto, K.: Volumetric modulated arc therapy: IMRT in a single gantry arc. Med. Phys. 35, 310-317 (2008)

3. Yu, C. X.: Intensity-modulated arc therapy with dynamic multileaf collimation: An alternative to tomotherapy. Phys. Med. Biol. 40, 1435-1449 (1995)

4. Yang, Y., Zhang, P., Happersett, L., Xiong, J., Yang, J., Chan, M., Beal, K., Mageras, G., Hunt, M.: Choreographing couch and collimator in volumetric modulated arc therapy. Int. J. Radiat. Oncol. Biol. Phys. 80, 1238-1247 (2011)

5. MacDonald, R. L., Thomas, C. G.: Dynamic trajectory-based couch motion for improvement of radiation therapy trajectories in cranial SRT. Med. Phys. 42, 2317$2325(2015)$

6. Smyth, G., Bamber, J. C., Evans, P. M., Bedford, J. L.: Trajectory optimisation for dynamic couch rotation during volumetric modulated arc radiotherapy. Phys. Med. Biol. 58, 8163-8177 (2013)

7. Rocha, H., Dias, J., Ferreira, B.C., Lopes, M.C.: Selection of intensity modulated radiation therapy treatment beam directions using radial basis functions within a pattern search methods framework. J. Glob. Optim. 57, 1065-1089 (2013)

8. Rocha, H., Dias, J., Ferreira, B.C., Lopes, M.C.: Beam angle optimization for intensity-modulated radiation therapy using a guided pattern search method. Phys. Med. Biol. 58, 2939-2953 (2013)

9. Rocha, H., Dias, J., Ferreira, B.C., Lopes, M.C.: Pattern search methods framework for beam angle optimization in radiotherapy design. Appl. Math. Comput. 219, 10853-10865 (2013)

10. Dias, J., Rocha, H., Ferreira, B.C., Lopes, M.C.: A genetic algorithm with neural network fitness function evaluation for IMRT beam angle optimization. Cent. Eur. J. Oper. Res. 22, 431-455 (2014)

11. Dias, J., Rocha, H., Ferreira, B.C., Lopes, M.C.: Simulated annealing applied to IMRT beam angle optimization: A computational study. Phys. Med. 31, 747-756 (2015)

12. Rocha, H., Dias, J., Ventura, T., Ferreira, B.C., Lopes, M.C.: A derivative-free multistart framework for an automated noncoplanar beam angle optimization in IMRT. Med. Phys. 43, 5514-5526 (2016)

13. Rocha, H., Dias, J., Ventura, T., Ferreira, B.C., Lopes, M.C.: Beam angle optimization in IMRT: are we really optimizing what matters?. Int. Trans. Oper. Res. 26, 908-928 (2019)

14. Papp, D., Bortfeld, T., Unkelbach, J.: A modular approach to intensity-modulated arc therapy optimization with noncoplanar trajectories. Phys. Med. Biol. 60, 5179$5198(2015)$

15. Langhans, M., Unkelbach, J., Bortfeld, T., Craft, D.: Optimizing highly noncoplanar VMAT trajectories: the NoVo method. Phys. Med. Biol. 63025023 (2018) 